\title{
Course keeping Control of Boeing 747 by using a Minimum-Order Observer State Controller
}

\author{
Nenad Popovich
}

\begin{abstract}
In this paper, a state space mathematical model of the Boeing 747 was defined, particularly for the lateral aircraft motions. A dynamical behavior of the model has been analized through numerical and graph-analytical methods. A Pole-placement approach of designing the control system was applied. A minimum-observer state based feedback controller was discussed. The whole model was developed by using Matlab and simulated by Simulink. A full-order observer was proposed because of its less sensitivity on measurement errors than the minimum-order (or reduced-order) observer (or state estimator). In the extreme cases, where the output noise is a significant problem, then an application of Kalman filter was suggested.
\end{abstract}

Keywords - Boeing 747, course keeping control, poleplacement, minimum-order observer, state controller, Matlab, Simulink.

\section{Introduction}

There are not many papers dealing with the plane control systems, mainly because a full information about them is usually classified, especially military ones.

The Boeing 747 is a large wide-body commercial plane. The equations of the body motion for the Boeing 747 are of eighth order, but they can be separated (decoupled) into two fourth-order sets, one of them representing the perturbations in longitudinal and another one lateral motions. The longitudinal motion defines the altitude movements of a plane, where the control system is especially critical during a take-off or landing. On the other hand, the lateral motions consist of two main parameters: rolling $(\phi, p)$ and yawing $(r$, $\beta)$ movements, [7]. In this paper, only the lateral motion will be discussed.

The whole plane coordinates are shown on the Fig.1.

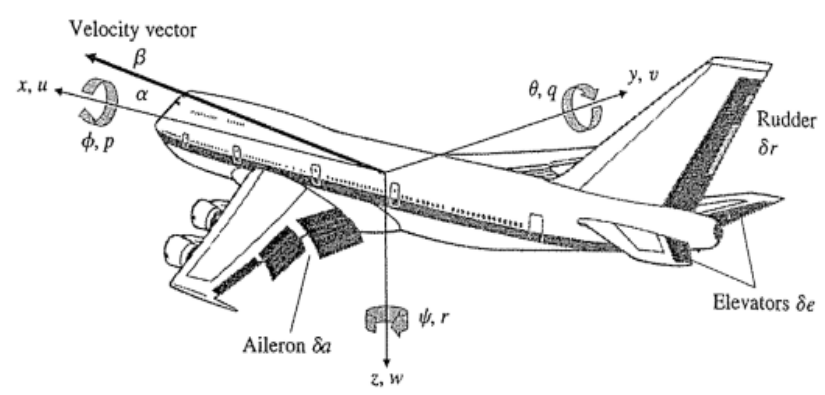

Fig.1. Definition of plane coordinates

\section{Nenad Popovich}

Auckland University of Technology, New Zealand where: where:

$$
\begin{aligned}
x, y, z & =\text { position coordinates } \\
u, v, w & =\text { velocity coordinates } \\
p & =\text { roll rate } \\
q & =\text { pitch rate } \\
r & =\text { yaw rate }
\end{aligned}
$$

$$
\begin{aligned}
\phi & =\text { roll angle } \\
\theta & =\text { pitch angle } \\
\psi & =\text { yaw angle } \\
\beta & =\text { side-slip angle } \\
\alpha & =\text { angle of attack }
\end{aligned}
$$

\section{Mathematical Model}

\section{A. Dynamical Equations}

Based on Fig.1, the non-linear equations of motions (dynamical model), including forces and moments that acting on the plane body, under proper assumptions [7], can be derived as in (1):

$$
\begin{gathered}
m(\dot{U}+q W-r V)=X-m g \sin \theta+\kappa T \cos \theta, \\
m(\dot{V}+r U-p W)=Y+m g \cos \theta \sin \phi, \\
m(\dot{W}+p V-q U)=Z+m g \cos \theta \cos \phi-\kappa T \sin \theta, \\
\quad I_{x} \dot{p}+I_{x z} \dot{r}+\left(I_{z}-I_{y}\right) q r+I_{x z} q p=L, \\
\quad I_{y} \dot{q}+\left(I_{x}-I_{z}\right) p r+I_{x z}\left(r^{2}-p^{2}\right)=M, \\
\text { (1) } I_{z} \dot{r}+I_{x z} \dot{p}+\left(I_{y}-I_{x}\right) q p-I_{x z} q r=N,
\end{gathered}
$$

Linearization of those equations can be carried out by neglecting some of nonlinear terms in (1), i.e. by assuming a small movements, as well as steady-state flight conditions with constant speed and attitude. Then, decouple two sets of equations (longitudinal and lateral, as mention before).

\section{B. State-Space Mathematical Model}

The modern trend in engineering systems is toward greater complexity, due mainly to the requirements of complex tasks and good accuracy. Complex systems, as it is an airplane, usually have multiple inputs and multiple outputs (MIMO), and may be time varying [1, 3, 4].

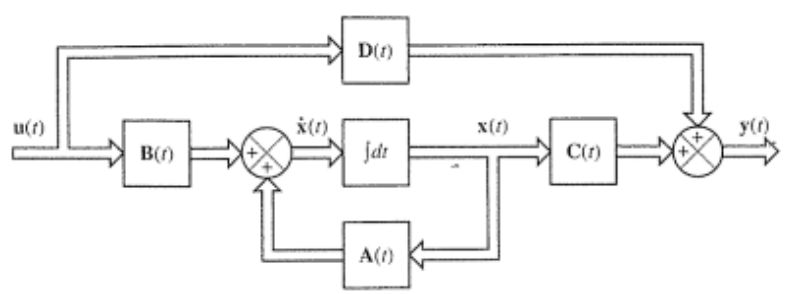

Fig.2. State space model in a general form

$$
\begin{aligned}
& \dot{x}=\mathbf{A x}+\mathbf{B u} \\
& \mathbf{y}=\mathbf{C x}+\mathbf{D u}
\end{aligned}
$$

where: 


$$
\begin{aligned}
& \mathbf{x}=\text { state vector }(n \text {-vector }) \\
& \mathbf{u}=\text { control vector }(r \text {-vector }) \\
& \mathbf{y}=\text { output vector }(m \text {-vector })
\end{aligned}
$$

$$
\begin{aligned}
& \mathbf{A}=n \times n \text { matrix } \\
& \mathbf{B}=n \times r \text { matrix } \\
& \mathbf{C}=m \times n \text { matrix } \\
& \mathbf{D}=m \times r \text { matrix }
\end{aligned}
$$

For linearized lateral motion of (1), the results are in the state-space form [7], as in (3):

$$
\left[\begin{array}{l}
\dot{\beta} \\
\dot{r} \\
\dot{p} \\
\dot{\phi}
\end{array}\right]=\left[\begin{array}{llll}
Y_{v} & -U_{0} & V_{0} & g_{0} \cos \theta_{0} \\
N_{v} & N_{r} & N_{p} & 0 \\
L_{v} & L_{r} & L_{p} & 0 \\
0 & \tan \theta_{0} & 1 & 0
\end{array}\right]\left[\begin{array}{l}
\beta \\
r \\
p \\
\phi
\end{array}\right]+\left[\begin{array}{l}
Y_{\delta} \\
N_{\delta} \\
L_{\delta} \\
0
\end{array}\right] \delta r
$$

In the case of lateral-perturbation equations of motion for Boeing 747 in horizontal flight at 40,000 ft and the nominal forward speed of $774 \mathrm{ft} / \mathrm{sec}$ (Mach 0.8), equation (3) becomes:

$$
\left[\begin{array}{l}
\dot{\beta} \\
\dot{r} \\
\dot{p} \\
\dot{\phi}
\end{array}\right]=\left[\begin{array}{llll}
-0.0558 & -0.9968 & 0.0802 & 0.0415 \\
0.598 & -0.115 & -0.0318 & 0 \\
-3.05 & 0.388 & -0.4650 & 0 \\
0 & 0.0805 & 1 & 0
\end{array}\right]\left[\begin{array}{l}
\beta \\
r \\
p \\
\phi
\end{array}\right]+\left[\begin{array}{l}
0.00729 \\
-0.475 \\
0.153 \\
0
\end{array}\right] \delta r
$$$$
y=\left[\begin{array}{llll}
0 & 1 & 0 & 0
\end{array}\right]\left[\begin{array}{l}
\beta \\
r \\
p \\
\phi
\end{array}\right]
$$

Note: Rudder $\left(\delta_{r}\right)$ is the input and yaw-rate $(r)$ is the output of the system.

In the traditional control, system is usually represented by transfer function, which can be derived from the state space model (4) in the form as in (5):

$$
-0.475 \mathrm{~s}^{\wedge} 3+0.1939 \mathrm{~s}^{\wedge} 2-0.09812 \mathrm{~s}-0.05633
$$

$$
s^{\wedge} 4-0.2942 s^{\wedge} 3+0.78 s^{\wedge} 2-0.0487 s+0.005532
$$

\section{(5)}

From the transfer function (particularly from denominator), it can be seen that system itself is unstable, and of course, a response to the step function of such open loop system will be unstable, too.

However, before applying a negative feedback, as well as a controller to that system, it will be good trying to make some modification of the system for better understanding the system itself.

\section{Re-modifying System}

As stated before, the output of the system is yaw-rate (r), with dimension in degree per second, and input (rudder movement, $\delta_{r}$ ) in degree. For a better understanding, output could be modified to yaw angle (course of the plane) also in degree as the input.

The new modified system becomes fifth order with matrices shown in (6).

$$
\left[\begin{array}{l}
\dot{\psi} \\
\dot{\beta} \\
\dot{r_{2}} \\
p_{2} \\
\dot{\phi}
\end{array}\right]=\left[\begin{array}{lllll}
0 & 0 & 1 & 0 & 0 \\
0 & -0.0558 & -0.9968 & 0.0802 & 0.0415 \\
0 & 0.598 & -0.115 & -0.0318 & 0 \\
0 & -3.05 & 0.388 & -0.4650 & 0 \\
0 & 0 & 0.0805 & 1 & 0
\end{array}\right]\left[\begin{array}{l}
\psi \\
\beta \\
r \\
p \\
\phi
\end{array}\right]+\left[\begin{array}{l}
0 \\
0.00729 \\
-0.475 \\
0.153 \\
0
\end{array}\right] \delta r
$$

$$
y=\left[\begin{array}{lllll}
1 & 0 & 0 & 0 & 0
\end{array}\right]\left[\begin{array}{l}
\psi \\
\beta \\
r \\
p \\
\phi
\end{array}\right]
$$

Note: a new output is $\psi$ (yaw angle or course).

And, a new transfer (output over input), $[2,5]$ is in (7):

$7.772 \mathrm{e}-016 \mathrm{~s}^{\wedge} 4-0.475 \mathrm{~s}^{\wedge} 3-0.2479 \mathrm{~s}^{\wedge} 2-0.1187 \mathrm{~s}-0.05633$

$$
s^{\wedge} 5+0.6358 s^{\wedge} 4+0.9389 s^{\wedge} 3+0.5116 s^{\wedge} 2+0.003674 s
$$

Note: A very small number (7.772e-016) in the numerator gives a very big positive zero (6.2e012), which ultimately gives unstable pole in the closed loop system for any gain (it was checked by Root Locus method), which leads to unstable system.

Ignoring that small number and change the sign in the numerator (which indicates only different orientation of rudder and yaw angle), transfer function becomes:

$0.475 s^{\wedge} 3+0.2479 s^{\wedge} 2+0.1187 s+0.0563$

$s^{\wedge} 5+0.6358 s^{\wedge} 4+0.9389 s^{\wedge} 3+0.5116 s^{\wedge} 2+0.003674 s$

(8)

Transfer function (8) has five poles and three zeros (9):

$$
\begin{aligned}
\multicolumn{1}{l}{\text { Poles: }} & \text { Zeros: } \\
0.0000+0.0000 i & \\
-0.0330+0.9467 i & \\
-0.0330-0.9467 i & -0.4980+0.0000 i \\
-0.5626+0.0000 i & -0.0119+0.4877 i \\
-0.0073+0.0000 i & -0.0119-0.4877 i
\end{aligned}
$$

Root Locus (checked, but not shown in this paper) indicates that system is always stable for any given gain.

Poles have an acceptable values, but their damping ratio (damping factor) is very small $(\zeta=0.03)$, which is far short of requirements for a good control $(\zeta \geq 0.5)$.

Specifications for the acceptable plane control conditions are:

natural frequency, $\omega_{\mathrm{n}} \leq 0.5$ and damping ratio, $\zeta \geq 0.5$.

Aircrafts with dynamics that violate those guidelines are generally considered fatiguing to fly and highly undesirable. Thus, our system specifications are to achieve lateral dynamics that meet those conditions. 


\section{Selecting the right Poles}

In addition of the above mentioned specifications, we would like that aircraft has also a good response characteristics, with:

- Overshoot less than $10 \%$

- Rise time less than 10 seconds

- $\quad$ Settling time less than 20 seconds

- Steady-state error less than $2 \%$

Based on natural frequency, $\omega_{\mathrm{n}}=0.5$, as well as a damping ration, $\zeta=0.7$ and by using "standard" formula (10), it is possible to calculate two dominant poles $[1,3,4]$.

$$
\frac{\omega_{n}^{2}}{s^{2}+2 \varsigma \omega_{n} s+\omega_{n}^{2}}
$$

Desired (dominant) poles are complex poles:

$$
\begin{aligned}
& \text { p1 }=-0.35+0.357 i \\
& \text { p2 }=-0.35-0.357 i
\end{aligned}
$$

Because our system is the fifth order, thus we need to derive additional three poles. We could also try to introduce a pole that cancels one of our zeros $(\mathrm{p} 3=-0.498)$, which will not be so close to the imaginary axis (i.e. non-dominant pole).

Same for another two poles, they need to be placed further away from the dominant poles, as well as on the LHS (Left Hand Side of the "s-plane" to avoid affecting the stability of the system. After a few randomly chosen those two poles, we found that the best dynamical behaviour of the system is with a double real pole $(\mathrm{p} 4=\mathrm{p} 5)$, which does not introduce extra oscillation in the system:

$$
\mathrm{p} 4=\mathrm{p} 5=-1
$$

\section{Design of Control System in State Space}

\section{A. Pole-Placement (Pole-Assignment)}

One of designing methods applied in this paper is, so called the Pole-placement or Pole-assignment technique. We assume that all state variables are measurable and are available for feedback. For checking those requirements, the system has to be completely observable (observability matrix,

$\mathrm{O}_{\text {obsv }}$ ) and completely state controllable (controllability matrix, $\left.\mathrm{C}_{\mathrm{ctrb}}\right),[4]$ :

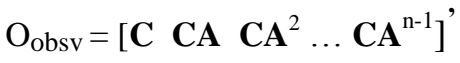

$$
\begin{aligned}
& \mathrm{C}_{\mathrm{ctrb}}=\left[\begin{array}{lllll}
\mathbf{B} & \mathbf{A} \mathbf{B} & \ldots & \mathbf{A}^{\mathrm{n}-1} \mathbf{B}
\end{array}\right]
\end{aligned}
$$

Both of those matrices have rank 5 (as it is our order of the modify system), which guarantees both: observability and controllability of the system. In that case, we can place our closed-loop poles at any desired locations by means of state feedback through an appropriate gain matrix, K.

Then, our state-space model can be transformed as in Fig.3:

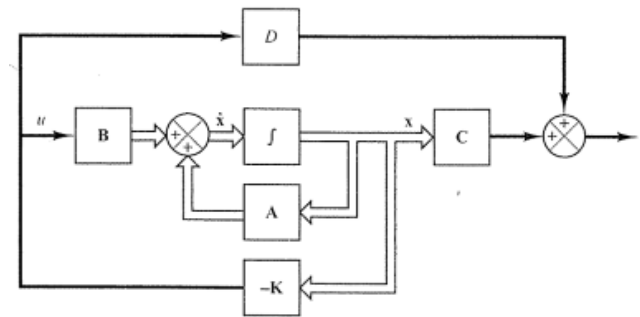

Fig.3 State-space model with feedback gain matrix (K)

In the conventional approach to the design of a single input single output (SISO) control system, only dominant closed-loop poles have desired $\omega \mathrm{n}$ and $\zeta$. The poleplacement approach specifies all closed-loop poles and because of that requires successful measurement of all state variables, or else requires the inclusion of the state observer (or the state estimator), which will be discussed later on.

Note: Control signal is $\mathrm{u}=-\mathrm{Kx}$. That means that closed loop system has no input and its objective is to maintain zero output.

$$
\dot{\mathbf{x}}(t)=(\mathbf{A}-\mathbf{B K}) \mathbf{x}(t)
$$

The stability and transient response characteristics are determined by the eigenvalues of (A-BK), [3, 4].

Note: eigenvalues are called in state space model (time domain) and poles in transfer function model (frequency domain), but the values are the same.

The pole-placement can be solved with Matlab by using "Ackerman formula" and its instruction ("acker")

$$
\begin{aligned}
& \mathbf{K}=\operatorname{acker}(\mathbf{A}, \mathbf{B},[\mathrm{p} 1 \mathrm{p} 2 \mathrm{p} 3 \mathrm{p} 4 \mathrm{p} 5]) \\
& \mathbf{K}=\left[\begin{array}{llll}
-2.2105 & 1.2275 & -5.4935 & -0.3671
\end{array}\right. \\
& (14)
\end{aligned}
$$

By using a linear simulation model, the output is shown on Fig.4:

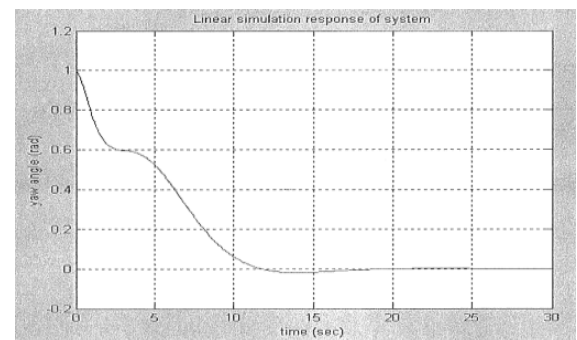

Fig.4 Output with state feedback gain matrix, $\mathbf{K}$

As we can see from the linear response graph (above), output (yaw angle, $\psi$ ) met all our criteria: with small overshoot, relatively small settling time, as well as no steady-state error (finishes in zero).

Note: the output starts from value 1 , because we set initial values: $\quad \mathbf{x}(0)=\left[\begin{array}{lllll}1 & 0 & 0 & 0 & 0\end{array}\right]$ ' 


\section{B. Observer Design}

As it was mentioned before, a pole-placement approach assumes that all state variables are available for feedback. However, in practice, it is not the case. Then, we need to estimate those inaccessible state variables. A device (or a computer programme) that estimates or observes the state variable is called a state observer, or simply an observer.

If the observer observes all variables, then it is called a fullstate observer. On the other hand, if observer observes some of state variables, then it is a reduced-order observer. A minimum-order observer observes only minimum possible variables, $[4,8,9]$.

\section{Minimum-Order Observer}

If only yaw angle $(\psi)$ is accessible for measurement, then

we have to estimate (observe) all other state variables for a feedback state controller. In that case Fig.3 has to be replaced, as it is shown on Fig.5.

Then, the state vector $(\mathbf{x})$ has to be partitioned into $\mathbf{x}_{\mathbf{a}}$ and $\mathbf{x}_{\mathbf{b}}$, since state variable $\mathbf{x}_{\mathbf{a}}$ is equal to output $\mathrm{y}$, which can be directly measured, and $\mathbf{x}_{\mathbf{b}}$ is the un-measured state vector:

$$
\begin{aligned}
& \quad y=\left[\begin{array}{ll}
1 & 0
\end{array}\left[\begin{array}{l}
x_{a} \\
x_{b}
\end{array}\right]\right. \\
& A_{a a}=\text { scalar } \\
& A_{a b}=1 \times(n-1) \text { matrix } \\
& A_{b a}=(n-1) \times 1 \text { matrix } \\
& A_{b b}=(n-1) \times(n-1) \text { matrix } \\
& B_{a}=s c a l a r \\
& B_{b}=(n-1) \times 1 \text { matrix }
\end{aligned}
$$$$
\left[\begin{array}{c}
\dot{x}_{a} \\
\dot{x}_{b}
\end{array}\right]=\left[\begin{array}{ll}
A_{a a} & A_{a b} \\
A_{b a} & A_{b b}
\end{array}\right]\left[\begin{array}{l}
x_{a} \\
x_{b}
\end{array}\right]+\left[\begin{array}{l}
B_{a} \\
B_{b}
\end{array}\right] u
$$

Comparing those matrices with (6), yield [5]:

$$
\begin{aligned}
A_{a a} & =0 \\
A_{a b} & =\left[\begin{array}{llll}
0 & 1 & 0 & 0
\end{array}\right] \\
A_{b a} & =\left[\begin{array}{l}
0 \\
0 \\
0 \\
0
\end{array}\right] \\
A_{b b} & =\left[\begin{array}{llll}
-0.0558 & -0.9968 & 0.0802 & 0.0415 \\
0.598 & -0.115 & -0.0318 & 0 \\
-3.05 & 0.388 & -0.465 & 0 \\
0 & 0.0805 & 1 & 0
\end{array}\right] \\
B_{a} & =0 \\
B_{b} & =\left[\begin{array}{llll}
0.0073 & -0.475 & 0.153 & 0
\end{array}\right],
\end{aligned}
$$

And, by performing some necessary transformations:

$$
\begin{aligned}
& \hat{A}=A_{b b}-K_{e} A_{a b} \\
& \hat{B}=\hat{A} K_{e}+A_{b a}-K_{e} A_{a a} \\
& \hat{F}=B_{b}-K_{e} B_{a} \\
& \hat{C}=\left[\begin{array}{l}
0 \\
I_{n-1}
\end{array}\right] \quad \hat{D}=\left[\begin{array}{l}
1 \\
K_{e}
\end{array}\right]
\end{aligned}
$$

As well as, previously found the gain matrix $\mathbf{K}$, partitioned:
$K_{a}=-2.2105$

$K_{b}=\left[\begin{array}{llll}1.2275 & -5.4935 & -0.3671 & -0.2063\end{array}\right]$

With all above transformations, our model becomes (Fig.5):

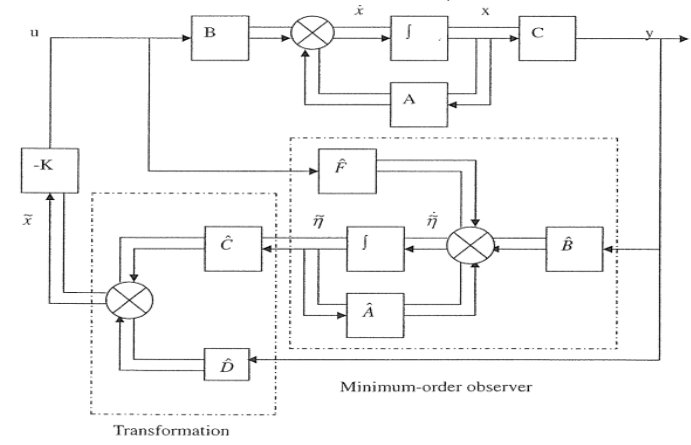

Fig.5 System with a minimum-order observer state feedback.

\section{Finding Minimum-Observer Gain Matrix $\left(K_{e}\right)$}

The error equation for the minimum-order observer, [4]:

$$
\dot{\mathbf{e}}=\left(\mathbf{A}_{b b}-\mathbf{K}_{c} \mathbf{A}_{a b}\right) \mathbf{e}
$$

If expressing state vector dynamics (12) and error dynamics (15) into one (augmented, 2 n order) matrix form, then:

(16)

$$
\left[\begin{array}{l}
\dot{x} \\
\dot{e}
\end{array}\right]=\left[\begin{array}{ll}
A-B K & B K_{b} \\
0 & A_{b b}-K_{e} A_{a b}
\end{array}\right]\left[\begin{array}{l}
x \\
e
\end{array}\right]
$$

As a general rule, the error dynamics has to be 2-5 times faster than controller's dynamics. That means: its poles have to be selected 2-5 times bigger than controller's poles, to make sure that observation error converges to zero quickly. If selected poles are 2 times faster:

$$
\begin{aligned}
& \mathrm{L}=\left[\begin{array}{llll}
-0.70+0.7142 \mathrm{i} & -0.70-0.7142 \mathrm{i} & -0.99 & -2.00
\end{array}\right] \\
& \mathbf{K}_{\mathbf{e}}=\operatorname{acker}\left(\mathrm{Abb}^{\prime}, \mathrm{Aab}^{\prime}, \mathrm{L}\right)^{\prime}=\left[\begin{array}{llll}
5.47 & 3.76 & -32 & 148
\end{array}\right]
\end{aligned}
$$

Then, by performing linear simulation, state variables and errors graphs are in Fig.6:

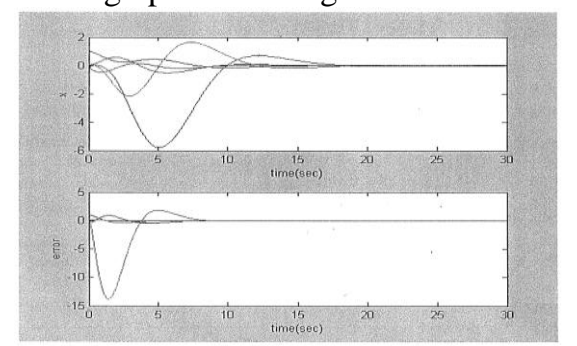

Fig.6 State variables (x) and errors (e) response ( 2 times faster observer poles)

If selected poles are 5 times faster:

$$
\begin{aligned}
& \mathrm{L}=\left[\begin{array}{llll}
-1.75+1.75 \mathrm{i} & -1.75-1.75 \mathrm{i} & -2.49 & -5.00
\end{array}\right] \\
& \mathbf{K}_{\mathbf{e}}=\operatorname{acker}\left(\mathrm{Abb},{ }^{\prime}, \mathrm{Aab}, \mathrm{L}\right){ }^{\prime}=\left[\begin{array}{llll}
-53 & 10 & -2225 & 8464
\end{array}\right]
\end{aligned}
$$

For selecting 5 times faster observer's poles, responses for errors are much faster, but the other hand, the amplitudes for 
errors (especially at the beginning) are very high due to very high gain constants in $\mathbf{K}_{\mathbf{e}}$, (Fig.7):

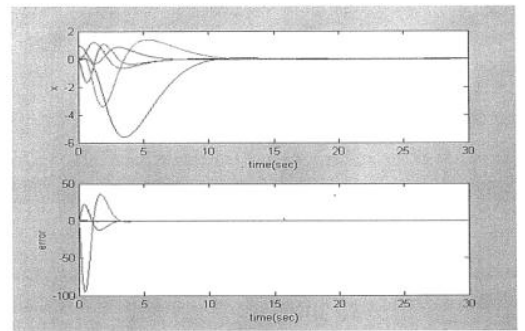

Fig.7 State variables (x) and errors (e) response (5 times faster observer poles)

A complete Simulink block diagram [5, 8] for the course keeping control system with a minimum-order observer is on the Fig.8, as well and all relevant responses in Fig.9.

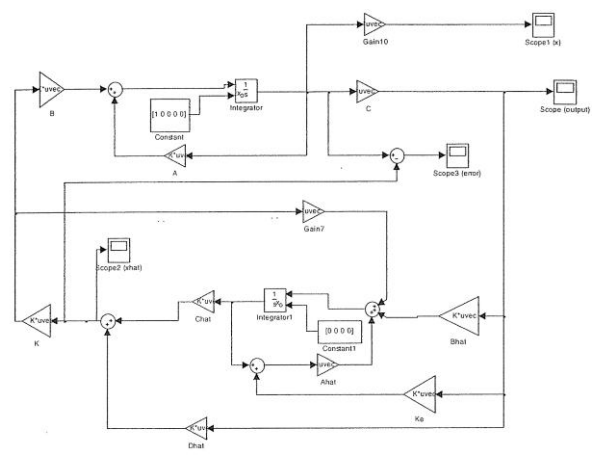

Simulink diagram for the whole system
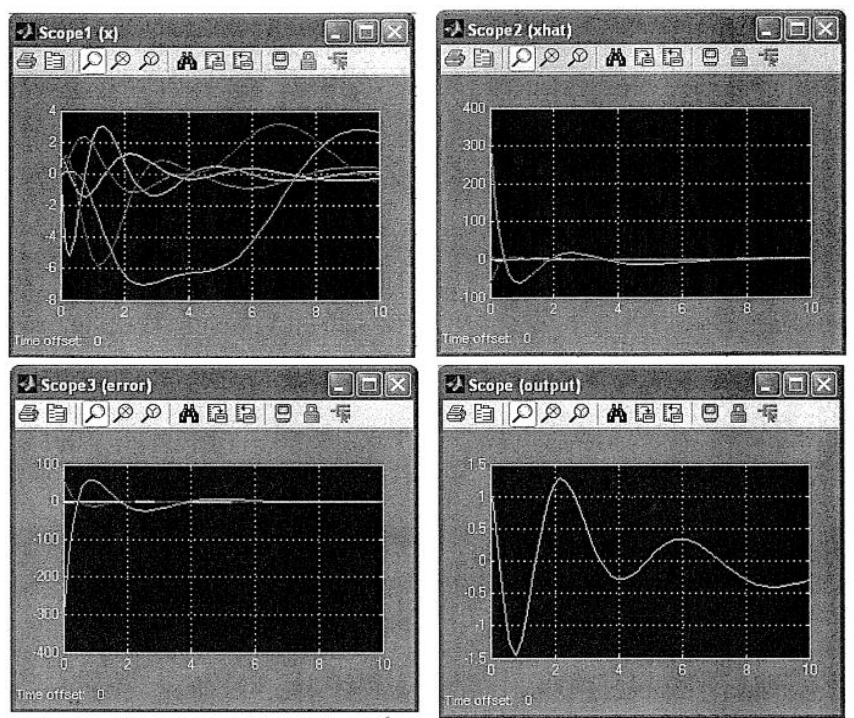

Fig.9 Responses for $\mathbf{x}, \mathbf{x h a t}$, errors (e) and output (y)

From those responses, it can be seen that although we set initial value only for yaw angle, all five state variables are affected (they are intertwined). In addition, the observer gives reasonable estimation for $\mathbf{x}$, as well as for $\mathbf{e}$ and $\mathbf{y}$.

\section{Conclusion}

Based on mathematical model in the state space and the transfer function, this paper analysed dynamics of the Boeing 747 aircraft, especially a lateral motion. The original model was re-modified and the proper selection of the poles found.
Based of desired dynamics, by using Pole-placement method, a feedback gain matrix $(\mathbf{K})$ was defined. A minimum-order observer was applied to the system to estimate immeasurable state variables. An observer gain matrix $\left(\mathbf{K}_{\mathbf{e}}\right)$ has been calculated by using Ackerman formula. The whole system:

aircraft, controller and minimum-order observer has been simulated by Simulink. The responses for state variables (x), observer variables (xhat), errors (e) and output (y) are shown on the separate graphs. Although, fast observer dynamics and high feedback gain would seem to give us all we asked for, such a combinations is sensitive to noise. In that case, a usage of less sensitive full-order observer or even Kalman filter is suggested.

\section{Acknowledgment}

I would like to express my gratitude to my final year project students, J.W Chang, for performing simulations with Matlab and Simulink.

\section{References}

[1] N.S. Nise, "Control Systems Engineering", Wiley, 7 ed, 2015.

[2] N. Popovich, P. Yan, "Determination of Q \& R Matrices for Optimal Pitch Aircraft", World Academy of Science \& Technology, pp.917923, 2011.

[3] R.C. Dorf, R.H. Bishop, "Modern Control Systems", Prentice Hall, $12^{\text {th }}$ ed., 2011.

[4] K. Ogata, "Modern Control Engineering", Prentice Hall, $5^{\text {th }}$ ed., 2010.

[5] J.W. Chang, "Control System: Lateral Control of Boeing 747”, Final year student's project (supervisor N.Popovich), 2005.

[6] N. Popovich, "Synthesis of the Ship Automatic Steering Control System", proceedings of Int. AMSE Conf. "Modelling \& Simuletions", Istanbul, Turkey, AMSE Press, Vol4a, pp. 141-148, 1988.

[7] G.F. Franklin, J.D. Powell, and A. Emanmi-Naeini, "Feedback Control of Dynamic Systems", Addison-Wesley Publiching Co., $3^{\text {rd }}$ ed., 1994

[8] N. Popovich, "Conception of the Ship Steering Control System with Observer State Feedback Controller", Int. AMSE Conf. "Signal, Data, Systems, with emphasis on Electrical \& Electronics Engg", Djerba, Tunisia, AMSE Press, Vol.1, pp. 291-296, 1991.

[9] D.G. Luenberger, "Observers for Multivariable Systems", IEEE, 1966.

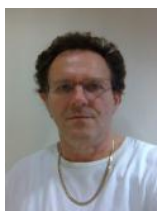

Nenad Popovich M.Sc. Engg (Hons) was born in Croatia in 1955. He graduated at the University of Zagreb, Croatia in 1978 and earned M.Sc. Degree in Engg. (Major in Control Systems) in 1984 at the same University. He has worked at five Universities. Currently, he is a senior lecturer at the Auckland University of Technology, New Zealand. He has twenty-two International conference and journal papers, as well as eight scientific research projects. 